\title{
Anti-inflammatory and Angiotensin I-Converting Enzyme Inhibitory of Malvidin and its Glycosides from Vaccinium Ashei
}

\author{
Han WU, Zheng YAN and Wu-yang HUANG ${ }^{a,{ }^{*}}$ \\ Institute of Farm Product Processing, Jiangsu Academy of Agricultural Sciences, \\ Nanjing, Jiangsu, PR China 210014 \\ awuyanghuang@hotmail.com \\ ${ }^{*}$ Corresponding author
}

Keywords: Blueberry, Malvidin and glycosides, Anti-inflammation, ACE inhibitor, Endothelial cells.

\begin{abstract}
Protective effects of malvidin and the glycosides from Vaccinium ashei on endothelial cells were investigated. The results showed that malvidin, malvidin-3-glucoside, malvidin-3-galactoside, and their mixture could defend endothelial cells from damage caused by TNF- $\alpha$, and inhibit monocyte chemotactic protein-1, intercellular adhesion molecule-1, vascular cell adhesion molecule-1, and angiotensin I-converting enzyme expression. In addition, they could inhibit degradation of I $\mathrm{B} \alpha$ and obstruct the nuclear translocation of p65, suggesting the anti-inflammatory mechanism of NF-кB pathway. The results indicated blueberry anthocyanins could be potential inflammation and ACE inhibitors, and blueberry could be functional foods advantageous to maintain a healthy cardiovascular population.
\end{abstract}

\section{Introduction}

Many fruits and vegetables may have benefit to human cardiovascular health [1]. Rabbiteye blueberry (Vaccinium ashei) is a "super" fruit, which possesses extensive pharmacological activities, e.g., antioxidant capacity, anti-diabetic effect, anti-proliferative activity, anti-inflammatory quality, and endothelial function improvement [2,3]. These health benefits mainly attribute to anthocyanins in blueberries [4,5]. Previous reports demonstrated that malvidin is the major anthocyanin in blueberries, which mainly exists as glycoside form, such as malvidin-3-glucoside and malvidin-3-galactoside (Fig. 1) [6,7]. These anthocyanins are great antioxidants.
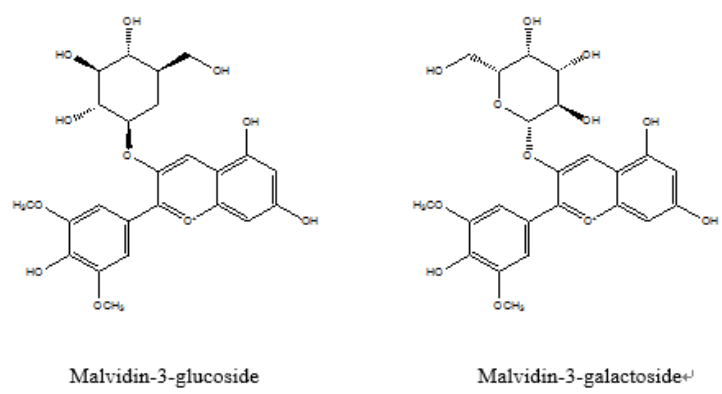

Fig. 1. Chemical structure of malvidin-3-glucoside and malvidin-3-galactoside.

As is known to all, endothelial function could predict cardiovascular outcomes [8]. Angiotensin I-converting enzyme (ACE) on the surface of the vascular endothelium, catalyzes angiotensin I (AngI) converse to a potent vasoconstrictor AngII, and inactivates a vasodilatory peptide bradykinin, thus it plays an important role in 
regulating blood pressure and cardiovascular function [9]. The vascular inflammatory response also plays a critical role in formation of cardiovascular diseases [10]. Endothelial cell activation is the major vector in vascular inflammation, which will increase circulating monocytes bonding to the wounded endothelial layer, and induce monocyte chemotactic protein-1 (MCP-1), intercellular adhesion molecule-1 (ICAM-1), and vascular cell adhesion molecule-1 (VCAM-1) expression [11,12]. Inflammatory cells accumulate reactive oxygen and nitrogen species, therefore, antioxidants could be potential to apply in prevention and treatment of chronic inflammation in many diseases, including cardiovascular disease [13]. In the present study, we investigated the influence of malvidin, malvidin-3-glucoside, malvidin-3-galactoside, and the mixture of two glycosides on endothelial cells, including pro-inflammatory cytokines MCP-1, ICAM-1, and VCAM-1, as well as ACE change.

\section{Materials and Methods}

\section{Chemicals and Reagents}

Dulbecco's phosphate buffered saline (PBS), M199 medium, tumor necrosis factor-alpha (TNF- $\alpha$ ), trypsin, malvidin (Mv), malvidin-3-glucoside (Mv-3-glc), and malvidin-3-galactoside (Mv-3-gal) were got from Sigma (Nanjing, China). Fetal bovine serum (FBS) was obtained from Gibco/Invitrogen (Shanghai, China). Penicillin and streptomycin were bought from Life Technologies (Shanghai, China). MCP-1, ICAM-1, VCAM-1, ACE ELISA Kit and ACE Enzyme Immunoassay Kit were purchased from Boster Biotechnology Inc. (Wuhan, China). ACE activation Assay Kit was bought from Beyotime Technology Inc. (Nanjing, China). PrimeScript RT master mix, Trizol reagent, and SYBR Green 2-step qRT-PCR kit were bought from TaKaRa Bio Inc. (Dalian, China). Nuclear factor-kappa $\mathrm{B}(\mathrm{NF}-\mathrm{\kappa B})$ activation and nuclear translocation Assay Kit were got from Beyotine Technology Inc. (Nanjing, China). All chemicals were of analytical-reagent grade.

\section{Antibodies}

Rabbit polyclonal primary antibody against VCAM-1, rabbit monoclonal primary antibody against ICAM-1, and mouse polyclonal primary antibody to the $\beta$-Actin, goat anti-rabbit/anti-mouse HRP-conjugated secondary antibody were got from Boster Biotechnology Inc. (Wuhan, China). Rabbit monoclonal primary antibody to I $\mathrm{B} \alpha$ was got from Beyotine Technology Inc. (Nanjing, China). Primary antibodies and secondary antibodies were used at 1:200 and 1:1000 dilutions, respectively.

\section{Endothelial Cell Culture and Treatment}

Human umbilical vein endothelial cells (HUVECs) were obtained from National Technical Research Centre of Veterinary Biological Products (Nanjing, China). HUVECs of the 2 to $6^{\text {th }}$ passage at $80-90 \%$ confluence were quiesced in a reduced serum medium for $4 \mathrm{~h}$, then treated with $10 \mu \mathrm{M} \mathrm{Mv}, \mathrm{Mv}-3$-glc, Mv-3-gal, (Mv-3-glc + Mv-3-gal) for $18 \mathrm{~h}$ prior to TNF- $\alpha(10 \mu \mathrm{g} / \mathrm{L})$ stimulation for $6 \mathrm{~h}$, respectively. The control used DMSO [14].

\section{Cell Vitality Detection}

The cell vitality was determined by MTT method [15]. The cells were added $10 \mu \mathrm{L}$ $0.5 \%(5 \mathrm{mg} / \mathrm{mL})$ MTT and then continued to culture $4 \mathrm{~h}$. Cell pellet was dissolved by adding $100 \mu \mathrm{L}$ DMSO and shaking 10 min slowly. The absorbance of $490 \mathrm{~nm}$ was 
measured on a StatFax-2100 Microplate Reader (Awareness Technology Inc., Plam, FL). Media were used to adjust zero, and untreated cells were as the blank. The cell vitality was calculated as follows: Cell viability $(\%)=$ sample group OD value/blank group OD value $\times 100 \%$

\section{ELISA Analysis}

MCP-1, ICAM-1, VCAM-1, and ACE proteins in the supernatants and ACE enzyme activity in cells were tested using ELISA kits. The absorbance of $450 \mathrm{~nm}$ was measured on a StatFax-2100 Microplate Reader to determine protein levels. The absorbance of $340 \mathrm{~nm}$ and $405 \mathrm{~nm}$ were measured every minute at $37^{\circ} \mathrm{C}$ for 9 min to determine ACE enzyme activity.

\section{Western Blotting}

ICAM-1, VCAM-1, and I $\mathrm{B} \alpha$ protein expression was analyzed by western blotting performed on the HUVEC lysates. The loading control used $\beta$-Actin. Data were normalized by the antibody against $\beta$-Actin, which were expressed as fold change to the control (untreated cells).

\section{Real-time Quantitative PCR}

The total RNA was isolated and reverse-transcribed into cDNA. The qRT-PCR analysis was carried out by the LightCycler 480 (Roche Diagnostics Inc., Switzerland). The primers for amplification were followed: forward primer 5-GTTGTCCCAAAGAAGC TGTGA-3 and reverse primer 5-AATCCGAACCCACTTCTGC-3 for MCP-1; forward primer 5-CCACAGTCACCTATGGCAAC-3 and reverse primer 5-AGTGTC TCCTGGCTCTGGTT-3 for ICAM-1; forward primer 5-GAACAAGTCGATGCTGG AGA-3 and reverse primer 5-ACCAGGTCCTCCAAGTTCAC-3 for ACE; forward primer 5-TGGACTTCGAGCAAGAGATG-3 and reverse primer 5-GAAGGAAGGC TGGAAGAGTG-3 for $\beta$-Actin [14]. The reaction started with a denaturing at $94^{\circ} \mathrm{C}$ for $30 \mathrm{~s}$, then 40 cycles of $60^{\circ} \mathrm{C}$ for $20 \mathrm{~s}$, and at $65^{\circ} \mathrm{C}$ for $15 \mathrm{~s}$. Data was analyzed by $2^{-\Delta \Delta \mathrm{Ct}}$ method.

\section{Immunofluorescence}

HUVECs were incubated with primary antibody against p65 overnight after fixation in $3.75 \%$ paraformaldehyde and permeabilized treatment with $0.1 \%$ Triton-X-100. The secondary antibody conjugated with the stain FTTC was added. DAPI was used to stain the cell nuclei [14]. The HUVECs were visualized using an Axiovision 4 Fluorescent Microscope (Zeiss, Germany).

\section{Statistical Analysis}

All data were presented as mean value \pm standard deviation (SD) of triplicate determinations. Figures were got from Microsoft Excel 2007. The data were analyzed by SPSS 19.0 Statistical Software. P value $<0.05$ was considered as significant difference.

\section{Results and Discussion}

\section{Effects on Cell Viability}

TNF- $\alpha$ exerts multiple biological effects and contributes dramatically to cellular cytotoxicity [16]. As strong antioxidants, malvidin and its glycosides might protect 
endothelial cells by oxidation defense mechanism [17]. In this study, TNF- $\alpha$ inhibited $70.3 \%$ endothelial cell, and its cell vitality was significantly decreased to $29.7 \%$. Pretreatment with malvidin, malvidin-3-glucoside, malvidin-3-galactoside, and the glycosides mixture could effectively protect endothelial cells from the damage caused by TNF- $\alpha$. The cell vitalities of $10 \mu \mathrm{M} \mathrm{Mv}, \mathrm{Mv}-3$-glc, Mv-3-gal, and the mixture (Mv-3-glc + Mv-3-gal) were 91.9\%, 87.6\%, 69.9\%, and 66.5\%, respectively (Table 1). Malvidin could improve endothelial cell vitality than its glycosides, and malvidin-3-glucoside showed increased protective effect on endothelial cells than malvidin-3-galactoside. These results indicated that malvidin and its glycosides should be safe nutraceuticals applied for disease prevention and treatment.

Table 1. Effect of Mv, Mv-3-glc, Mv-3-gal, and the glycosides mixture on HUVEC cell viability and MCP-1, ICAM-1, and VCAM-1 induced by TNF- $\alpha$ in supernatant.

\begin{tabular}{cclcl}
\hline Treatment & Cell viability [\%] & \multicolumn{3}{c}{ Protein in supernatant $[\mu \mathrm{g} / \mathrm{L}]$} \\
\cline { 3 - 5 } & & \multicolumn{1}{c}{ MCP-1 } & \multicolumn{1}{c}{ ICAM-1 } & VCAM-1 \\
\hline Control & $100.0 * *$ & $0.183 \pm 0.007 * * *$ & $0.133 \pm 0.002^{* *}$ & $0.147 \pm 0.011^{* *}$ \\
TNF- $\alpha$ & $29.7 \pm 0.1$ & $0.608 \pm 0.018$ & $0.669 \pm 0.033$ & $0.538 \pm 0.019$ \\
Mv & $91.9 \pm 6.0 * *$ & $0.355 \pm 0.016 * * *$ & $0.258 \pm 0.019 * *$ & $0.244 \pm 0.009 * * *$ \\
Mv-3-glc & $87.6 \pm 3.0 * *$ & $0.437 \pm 0.016^{* *}$ & $0.537 \pm 0.095^{*}$ & $0.365 \pm 0.017 * *$ \\
Mv-3-gal & $69.9 \pm 1.4 * *$ & $0.468 \pm 0.019 * *$ & $0.384 \pm 0.048^{* *}$ & $0.389 \pm 0.018^{* *}$ \\
mixture & $66.5 \pm 2.5 * *$ & $0.229 \pm 0.014 * * *$ & $0.219 \pm 0.038^{* *}$ & $0.243 \pm 0.014 * * *$ \\
\hline
\end{tabular}

$*, * *$, and $* * *$ indicate $P<0.05, P<0.01$, and $P<0.001$ respectively compared to TNF- $\alpha$ group.

The effects on the TNF- $\alpha$-induced MCP-1, ICAM-1, and VCAM-1 in the cell-free endothelial supernatants were evaluated using ELISA, while proteins in cells were evaluated by western blotting. In un-stimulated cells, MCP-1, ICAM-1, and VCAM-1 protein expression was very low. The expression was strongly up-regulated when exposed to TNF- $\alpha$. Pretreatment with Mv, Mv-3-glc, Mv-3-gal, and the mixture could partially inhibit this up-regulation of MCP-1, ICAM-1, and VCAM-1 expression by TNF- $\alpha$ (Table 1). The inhibitory effect of Mv, Mv-3-glc, Mv-3-gal and (Mv-3-glc + Mv-3-gal) on MCP-1 induced by TNF- $\alpha$ was $59.5 \%, 40.2 \%, 32.9 \%$, and $89.2 \%$, respectively. In addition, they inhibited $76.7 \%, 24.6 \%, 53.2 \%$ and $83.9 \%$ of ICAM-1; and $75.2 \%, 44.2 \%, 38.1 \%$, and $75.4 \%$ of VCAM-1, respectively.

\section{Inflammation Inhibition}

Similar to supernatant, TNF- $\alpha$ significantly up-regulated ICAM-1 and VCAM-1 levels in cells, whereas $\mathrm{Mv}$ and its glycosides could decrease the TNF- $\alpha$-induced proteins scavenging activity (Fig. 2a). Malvidin could inhibit 44.9\% ICAM-1 and 69.3\% VCAM-1. Mv-3-glc inhibited 70.8\% ICAM-1 and 46.5\% VCAM-1, while Mv-3-gal inhibited 49.2\% ICAM-1 and 58.8\% VCAM-1. The mixture of Mv-3-glc and Mv-3-gal significantly inhibited $86.8 \%$ ICAM-1 and $76.2 \%$ VCAM-1, respectively. 


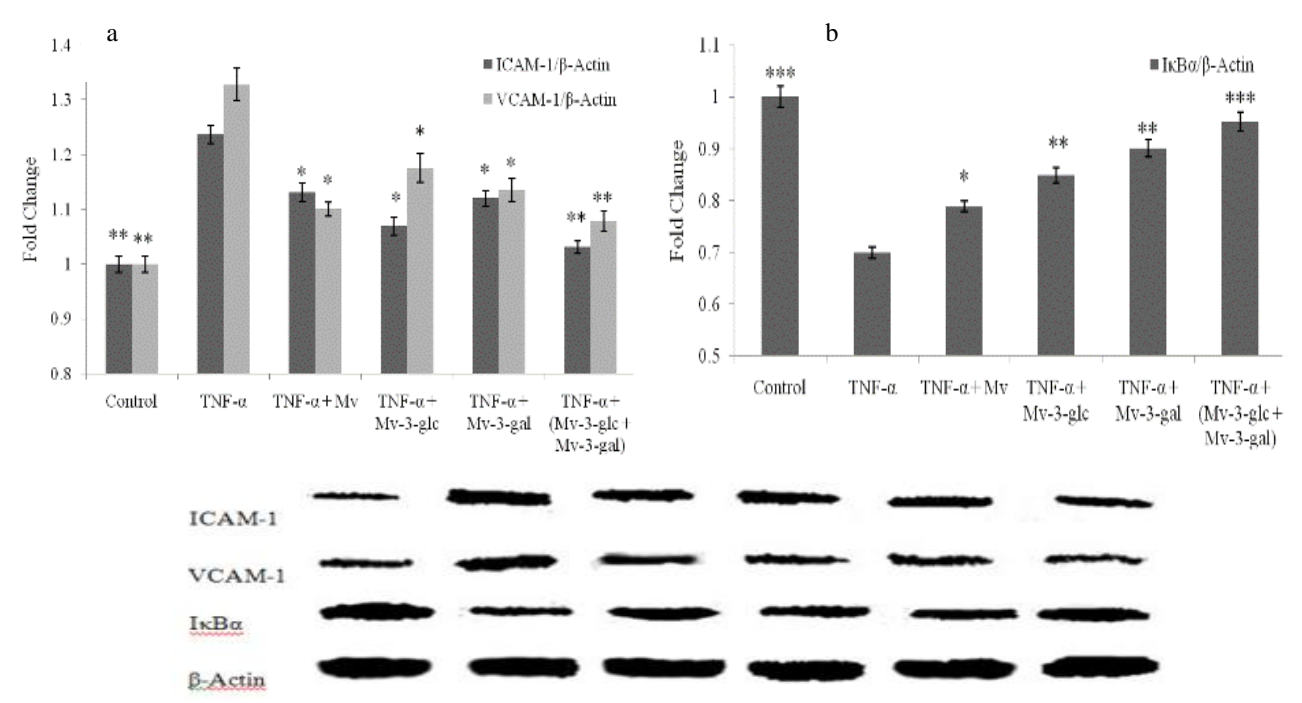

Fig. 2. Effect of Mv, Mv-3-glc, Mv-3-gal, and the glycosides mixture on TNF- $\alpha$-induced (a) ICAM-1 and VCAM-1, (b) IאB $\alpha$ protein expression in cells. Representative western blots are shown.

$*$, **, and $* * *$ indicate $\mathrm{P}<0.05, \mathrm{P}<0.01$ and $\mathrm{P}<0.001$ respectively compared to TNF- $\alpha$ alone group.

The MCP-1 and ICAM-1 mRNA expression levels were measured by reverse transcription PCR using $\beta$-Actin as internal standard. Data are expressed as fold change to the control. Figure 3 showed that the mRNA levels of MCP-1 and ICAM-1 in the cells were increased when cultured with TNF- $\alpha$ only. Pretreatment with Mv, Mv-3-glc, Mv-3-gal, and the mixture can inhibit $68.5 \%, 81.9 \%, 68.2 \%, 94.4 \%$ of MCP-1, and $82.3 \%, 37.6 \%, 43.4 \%, 89.2 \%$ of ICAM-1, respectively.

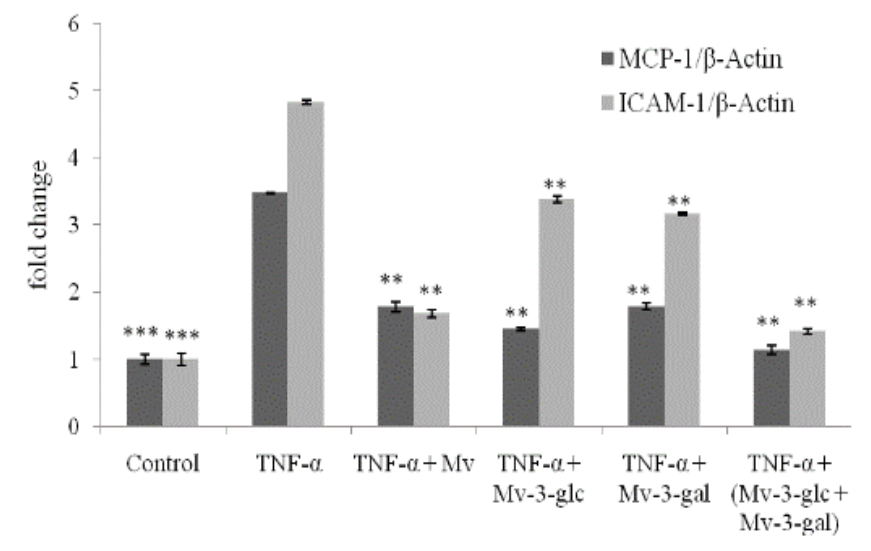

Fig. 3. Effect of Mv, Mv-3-glc, Mv-3-gal, and the glycosides mixture on TNF- $\alpha$-induced MCP-1 and ICAM-1mRNA expression.

** and *** indicate $\mathrm{P}<0.01$ and $\mathrm{P}<0.001$ respectively compared to TNF- $\alpha$ alone group.

The effect of malvidin and the two glycosides on anti-inflammation mechanism was detected using western blotting in the present study. The amount of I $\mathrm{K} B \alpha$ protein was greatly decreased when exposed to TNF- $\alpha$. The inhibition rates of Mv, Mv-3-glc, Mv-3-gal, and the mixture on I $\kappa \mathrm{B}$ degradation caused by TNF- $\alpha$ were $29.8 \%, 49.7 \%$, $66.7 \%$, and $84.3 \%$, respectively (Fig. $2 b$ ). The p65 subunit's translocation from the cytoplasm to the nucleus induces NF- $\kappa \mathrm{B}$ activation. Immunocytochemistry was investigated using fluorescein isothiocyanate (FITC)-conjugated antibody. The p65 level in the nucleus was low. After stimulation with TNF- $\alpha$, the nuclear p65 level was increased. However, the fluorescence intensity of p65 was decreased in the nucleus with 
the treatment of Mv, Mv-3-glc, Mv-3-gal, and the mixture (Fig. 4). As a result, the glycosides mix ture apparently blocked the NF- $\mathrm{NB}$ translocation.
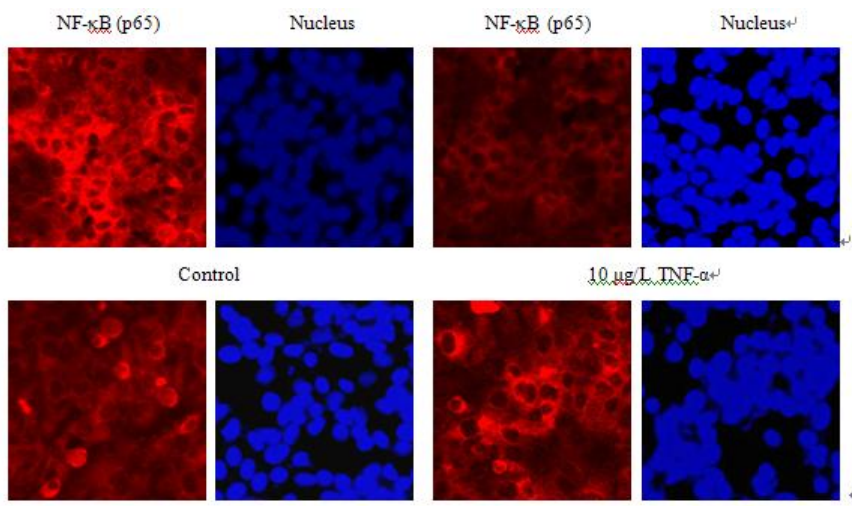

$10 \mathrm{MMMN}+\mathrm{TNF}-\alpha$

10 uld Mv-3-glc + TNF- $\alpha$
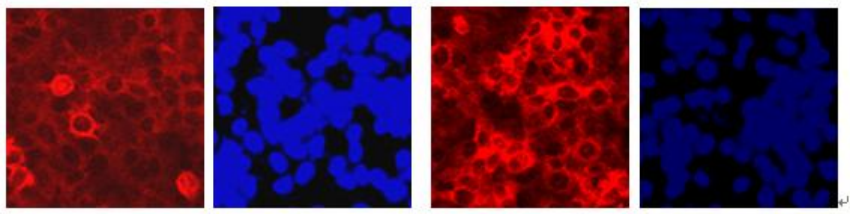

$10 \mathrm{MML}$ Mv-3-gal + TNF-a

$10 \mu \mathrm{M}(\mathrm{Mv}-3-\mathrm{gal}+\mathrm{Mv}-3-\mathrm{gal})+\mathrm{TNF}-\alpha$

Fig. 4. Effect of Mv, Mv-3-glc, Mv-3-gal, and the glycosides mixture on NF-kB pathway (endothelial p65 translocation). A representative set of images from 6 independent experiments are shown. All images presented are in X100 magnification.

The inhibitors of MCP-1, ICAM-1, and VCAM-1 represent potential development to treat inflammatory diseases as a novel strategy. MCP-1, ICAM-1, and VCAM-1 were over-expressed in endothelial cells exposed to TNF- $\alpha[12,18]$. This study demonstrates that treatments with $\mathrm{Mv}, \mathrm{Mv}-3$-glc, Mv-3-gal and the glycosides mixture significantly inhibited TNF- $\alpha$-induced MCP-1, ICAM-1, and VCAM-1 expression both in cell and cell-free endothelial supernatants from protein level and mRNA level. All the data showed the glycosides mixture possessed the strongest anti-inflammatory effects. In accordance with endothelial cell vitality, malvidin produce greater inhibition rate than its two glycosides in the most case, except for MCP-1 mRNA and ICAM-1 protein in cells. It might because glycosylation decreases the hydroxyl group which was most important factor in determining physiological function of anthocyanins [5]. In addition, the changes in the anti-inflammatory effect of $\mathrm{Mv}-3$-glc mostly were more pronounced than Mv-3-gal. Malvidin with the glycosides affected the levels of I $\mathrm{KB} \alpha$ and inhibitted the subunit of NF- $\kappa B$ (p65) on the nuclear translocation, suggesting the anti-inflammation mechanism by blocking the NF- $\kappa$ B pathway.

\section{Angiotensin I-converting Enzyme Inhibition}

ELISA results showed that ACE protein expression in the cell-free supernatants and ACE enzyme activity of endothelial cells were significantly increased by stimulation of TNF- $\alpha$. The ACE protein level was increased from 0.328 to $0.794 \mu \mathrm{g} / \mathrm{L}$, which was 2.4 fold of the control. ACE enzyme activity was from 4.6 to $12.7 \mathrm{U} / \mathrm{L}$, which was 2.7 fold of the control. Malvidin and its glycosides could greatly decrease ACE induced by TNF- $\alpha$. Malvidin, Mv-3-glc, Mv-3-gal, and (Mv-3-glc + Mv-3-gal) inhibited 28.3\%, $74.1 \%, 37.3 \%$, and $81.2 \%$ TNF- $\alpha$-increased ACE protein levels, and 58.9\%, 67.4\%, $64.3 \%$, and 78.5\% TNF- $\alpha$-increased ACE enzyme activity, respectively (Fig. 5a,b). 


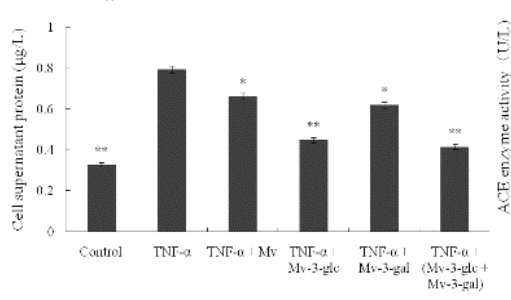

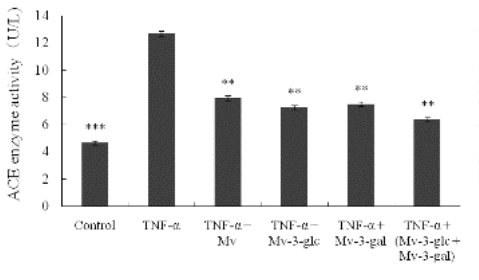

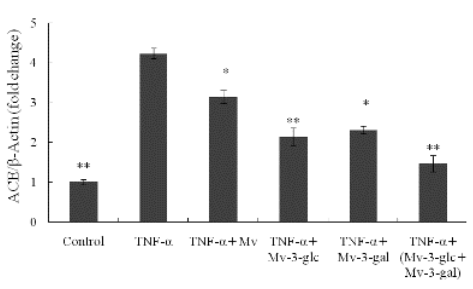

Fig. 5. Effect of Mv, Mv-3-glc, Mv-3-gal, and the glycosides mixture on TNF- $\alpha$-induced (a) ACE protein level in cell supernatant, (b) ACE enzyme activity, (c) ACE mRNA expression in HUVEC cells.

$*$, **, and ${ }^{* *}$ indicate $\mathrm{P}<0.05, \mathrm{P}<0.01$ and $\mathrm{P}<0.001$ respectively compared to TNF- $\alpha$ alone group.

Similarly, real-time qRT-PCR results showed that malvidin and its glycosides could effectively reduce the ACE mRNA levels. TNF- $\alpha$ group increased 3.2 fold change compared to the control. Malvidin, Mv-3-glc, Mv-3-gal, and (Mv-3-glc + Mv-3-gal) inhibited $33.5 \%, 64.8 \%, 59.4 \%$, and $85.7 \%$ TNF- $\alpha$-increased ACE mRNA expression, respectively (Fig. 5c). Although malvidin showed enhanced cell vitality than two malvidin glycosides, glucoside and galactoside seemed to improve ACE inhibitory effect in this study. Mv-3-glc also possessed better inhibition on ACE than Mv-3-gal. In addition, the mixture exhibited the greatest effect indicating the synergy effect between malvidin-3-glucoside and malvidin-3-galactoside.

ACE inhibitors are important antihypertensive drugs [1]. Blueberries rich in anthocyanins could be of benefit for hypertensive patients. Anthocyanins are considered the most potent hydrophilic antioxidants in nature and have benefits in cardiovascular health by improving endothelial function [19]. Lee and others previously reported that extract of purple barley grain and bran rich in anthocyanin inhibited ACE enzyme activity with half maximal inhibitory concentration $\left(\mathrm{IC}_{50}\right)$ of 8.77 and $4.54 \mathrm{mg} / \mathrm{mL}$, respectively [20]. In addition, Ojeda and others found that the anthocyanins of Hibiscus sabdariffa, delphinidin-3-sambubiosides and cyanidin-3-sambubiosides inhibited the ACE enzyme activity by competting the active site with the substrate [21]. This study found blueberry anthocyanins malvidin and its glycosides all could inhibit above 50\% TNF- $\alpha$-increased ACE enzyme activity in endothelial cells, which indicated they could be used as potential ACE inhibitors.

\section{Summary}

In conclusion, anthocyanins malvidin and its glycosides derived from blueberries could protect endothelial cells through potential anti-inflammatory and ACE inhibitory effects. Pretreatment with Mv, Mv-3-glc, Mv-3-gal, and the glycosides mixture significantly attenuated endothelial damage caused by TNF- $\alpha$ through inhibiting MCP-1, ICAM-1, VCAM-1, and ACE production both in levels of protein and mRNA expression, as well as ACE enzyme activity. This study demonstrated that malvidin and its glycosides attenuated TNF- $\alpha$ increased inflammatory responses in HUVECs by blocking the NF- $\mathrm{KB}$ pathway. Additionally, Mv-3-glc and Mv-3-gal had some synergistic effect on anti-inflammatory and ACE inhibitory activity. This indicated that blueberries are functional foods as a good anthocyanins resource of inflammation and ACE inhibitors, having potential to develop nutraceuticals for improving endothelial function and preventing the cardiovascular diseases. 


\section{Acknowledgement}

This research was financially supported by the Natural Science Foundation Program of Jiangsu Province (20161376) and the Scientific Researching Foundation Program of Institute of Farm Product Processing (013036611703).

\section{References}

[1] W.Y. Huang, S.T. Davidge, J. Wu. Bioactive natural constituents from food sources - potential use in hypertension prevention and treatment, Crit. Rev. Food Sci. 53 (2013) 615-630.

[2] J. Kanterman, M. Sade-Feldman, M. Baniyash. New insights into chronic inflammation-induced immunosuppressant, Semin. Cancer Biol. 22 (2012) 307-308.

[3] K.S. Shaughnessy, I.A. Boswall, A.P. Scanlan, K.T. Gottschall-Pass, M.I. Sweeney. Diets containing blueberry extract lower blood pressure in spontaneously hypertensive stroke-prone rats, Nutr. Res. 29 (2009) 130-138.

[4] A.D.R. Castrejon, I. Eichholz, S. Rohn, L.W. Krohn, S. Huykens-Keil. Phenolic profile and antioxidant activity of highbush blueberry (Vaccinium corymbosum L.) during fruit maturation and ripening, Food Chem. 109 (2008) 564-572.

[5] E. Pojer, F. Mattivi, D. Johnson, C.S. Stockley. The case for anthocyanin consumption to promote human health: A review, Compr. Rev. Food Sci. F. 12 (2013) 483-508.

[6] F.S. Hosseinian, T. Beta. Saskatoon and wild blueberries have higher anthocyanin contents than other Manitoban berries, J. Agr. Food Chem. 55 (2007) 10832-10838.

[7] C.Y. Li, J. Feng, W.Y. Huang, X.T. An. Composition of polyphenols and antioxidant activity of rabbiteye blueberry (Vaccinium ashei) in Nanjing, J. Agr. Food. Chem. 61 (2013) 523-531.

[8] Y. Higashi, K. Noma, M. Yoshizumi, Y. Kihara. Endothelial function and oxidative stress in cardiovascular diseases, Circ. J. 73 (2009) 411-418.

[9] S.Q. Rao, J. Sun, Y.J. Su, R.R Xu, Y.J Yang. Purification and characterization of angiotensin I-converting enzyme inhibitory peptides from enzymatic hydrolysate of hen egg white lysozyme, Food Res. Int. 46 (2012) 127-134.

[10] U. Landmesser, D.G. Harrison. Oxidant stress as a marker for cardiovascular events: Ox marks the spot, Circulation 104 (2001) 2638-2640.

[11] W. Bakker, E.C. Eringa, S.P. Ipkema, V.W. Hinsbergh. Endothelial dysfunction and diabetes: roles of hyperglycemia, impaired insulin signaling and obesity, Cell. Tissue. Res. 335 (2009) 165-189.

[12] L. Martin, W. Birger, J. Franziska, R. Christian, V. Dark, S. Alexander, B.D. Johannes, B.F. Stephan. Cerivastain reduces cytokine-induced surface expression of ICAM-1 via increased shedding in human endothelial cells, Atherosclerosis 190 (2007) 43-52.

[13] H.Y. Li, Z.Y. Deng, H.H. Zhu, C.L. Hu, R.H. Liu, J.C. Young, R. Tsao. Highly pigmented vegetables: Anthocyanin compositions and their role in antioxidant activities, Food Res. Int. 46 (2010) 250-259. 
[14] W.Y. Huang, Y.M. Liu, J. Wang, X.N. Wang, C.Y. Li. Anti-inflammatory effect of the blueberry anthocyanins malvidin-3-glucoside and malvidin-3-galactoside in endothelial cells, Molecules. 19 (2014) 12827-12841.

[15] C.T. Chang, M. Korivi, H.C. Huang, V. Thiyagarajan, K.Y. Lin, P.J. Huang, J.Y. Liu, Y.C. Hseu, H.L. Yang. Inhibition of ROS production, autophagy or apoptosis signaling reversed the anticancer properties of Antrodia salmonea in triple-negative breast cancer (MDA-MB-231) cells, Food. Chem. Toxicol. 103 (2017) 1-17.

[16] J. Weber, V. Tiriveedhi, M. Takenaka, W. Lu, R. Hachem, E. Trulock, G.A. Patterson, T. Mohanakumar. Inhibition of renin angiotensin aldosterone system causes abrogation of obliterative airways disease through inhibition of tumor necrosis factor- $\alpha$-dependant interleukin-17, J. Heart. Lung. Transpl. 31 (2012) 419-426.

[17] P. Joana, C.P.D. Teresa, M.A. Leonor. Malvidin-3-glucoside protects endothelial cells up-regulating endothelial NO synthase and inhibiting peroxynitrite-induced NF-kB activation, Chem-biol. Interact. 199 (2012) 192-200.

[18] L. Yang, R.M. Froio, T.E. Sciuto, A.M. Dvorak, R. Alon, F.W. Luscinskas. ICAM-1 regulates neutrophil adhesion and transcellular migration of TNF-alpha-activated vascular endothelium under flow, Blood, 106 (2005) 584-592.

[19] L.J. Wang, S. Su, J. Wu, H. Du, S.S. Li, J.W. Huo, Y. Zhang, L.S. Wang. Variation of anthocyanins and flavonols in Vaccinium uliginosum berry in Lesser Khingan Mountains and its antioxidant activity, Food. Chem. 160 (2014) 357-364.

[20] C. Lee, D. Han, B. Kim, N. Baek, B.K. Baik. Antioxidant and anti-hypertensive activity of anthocyanin-rich extracts from hulless pigmented barley cultivars, Int. J. Food Sci. Tech. 48 (2013) 984-991.

[21] D. Ojeda, E. Jiménez-Ferrer, A. Zamilpa, A. Herrera-Arellano, J. Tortoriello, L. Alvarez. Inhibition of angiotensin convertin enzyme (ACE) activity by the anthocyanins delphinidin- and cyanidin-3-O-sambubiosides from Hibiscus sabdariffa, J. Ethnopharmacol. 127 (2010) 7-10. 